\title{
Dosimetric comparison of tools for intensity modulated radiation therapy with gamma analysis: a phantom study
}

\author{
Ugur Akbas,a, Murat Okutan¹, Bayram Demir², Canan Koksal ${ }^{1}$ \\ ${ }^{1}$ Istanbul University, Oncology Institute, Department of Medical Physics, Istanbul, Turkey \\ ${ }^{2}$ Istanbul University, Science Faculty, Department of Physics, Istanbul, Turkey
}

\begin{abstract}
Dosimetry of the Intensity Modulated Radiation Therapy (IMRT) is very important because of the complex dose distributions. Diode arrays are the most common and practical measurement tools for clinical usage for IMRT. Phantom selection is critical for QA process. IMRT treatment plans are recalculated for the phantom irradiation in QA. Phantoms are made in different geometrical shapes to measure the doses of different types of irradiation techniques. Comparison of measured and calculated dose distributions for IMRT can be made by using gamma analysis. In this study, 10 head-and-neck IMRT QA plans were created with Varian Eclipse 8.9 treatment planning system. Water equivalent RW3-slab phantoms, Octavius-2 phantom and PTW Seven29 2D-array were used for QA measurements. Gantry, collimator and couch positions set to $0^{0}$ and QA plans were delivered to RW3 and Octavius phantoms. Then the positions set to original angles and QA plans irradiated again. Measured and calculated fluence maps were evaluated with gamma analysis for different DD and DTA criteria. The effect of different set-up conditions for RW3 and Octavius phantoms in QA plan delivery evaluated by gamma analysis. Results of gamma analysis show that using RW3-slab phantoms with setting parameters to $0^{0}$ is more appropriate for IMRT QA.
\end{abstract}

\section{Introduction}

Intensity modulated radiation therapy (IMRT) technique poses such challenges for measuring quality assurance (QA) of the complex dose distributions. Treatment plans that modulated by multi-leaf collimation lead to numerous regions containing steep dose gradients. For a proper IMRT implementation, comprehending the use of dosimetric tools to measure the doses is important.

Point dosimetry may allow validating the IMRT dose distributions at individual points, but quality assessment of modulated dose distributions requires two dimensional (2D) dosimetry at least. Diode arrays are the most common and practical measurement tools for clinical usage. Phantom selection is critical for QA process. The appropriate phantom should be determined by the purpose of measurement. Treatment planning systems calculate dose to patients in regard to constrains defined at optimization page. These plans are recalculated for the phantom irradiation in QA. For an accurate calculation, phantoms should be made of water-equivalent or known electron-density material. Phantoms are made in different geometrical shapes to measure the doses of different types of irradiation techniques [1].

Irradiation of tumor and simultaneous protection of the organs at risk is the main point of IMRT. Comparison of measured and calculated dose distributions for IMRT can be made by using gamma analysis. After creating dose fluence maps by measuring with dosimetric tool and by calculating with treatment planning system, the gamma method measures the closest distance between each reference point and evaluated dose distribution after scaling by DD (Dose-difference-criteria) and DTA (distance-to-agreement). The method provides an evaluation of either dosimetric or spatial disagreement.

\section{Materials and Methods}

\subsection{Treatment Planning}

Computed tomography (CT) images of 10 nasopharynx cancer patients were acquired in head gantry and supine position. Thickness of the CT images is $3 \mathrm{~mm}$. Gross tumor volume (GTV), clinical target volume (CTV), planning target volume (PTV) and organs at risk (OARs) were defined and contoured by radiation oncologist. Then images were sent to Varian Eclipse treatment planning system (TPS). 7 field IMRT plans were created for each patient. 6MV energy was used for each of 7 fields in Varian Trilogy linear accelerator (LINAC). Analytical anisotropic algorithm (AAA) was used for calculation. Calculation grid size was chosen $2.5 \mathrm{~mm}$. QA plans were created in some conditions. Gantry, collimator and couch positions set to $0^{0}$ and QA plans were created for RW3 and Octavius phantoms. Then the positions set to original angles and QA plans were created for RW3 and Octavius phantoms. Created QA plans were irradiated under these conditions.

a Corresponding author: u.akbas@yahoo.com 


\subsection{Measurements and Gamma Evaluation}

Irradiations for each condition were measured by using PTW Seven29 2D-array, which is an ion chamber array with 729 ion chambers for precise IMRT plan verification and LINAC QA. In Seven29, the vented plane-parallel ion chambers are $5 \mathrm{~mm} \times 5 \mathrm{~mm} \times 5 \mathrm{~mm}$ in size, and the center-to-center spacing is $10 \mathrm{~mm}$. In total there are located 729 chambers in a matrix of $27 \times 27$, providing a maximum field size of $27 \mathrm{~cm} \mathrm{x} 27 \mathrm{~cm}$. The array is only $22 \mathrm{~mm}$ flat and $3.2 \mathrm{~kg}$ light. The surrounding material is acrylic (PMMA) [2].

The gamma method provides an evaluation of either dosimetric or spatial disagreement with measuring the closest distance between each reference point and evaluated dose distribution after scaling by Dosedifference-criteria and distance-to-agreement. The concept of gamma verification is shown in Fig. 1 [3].

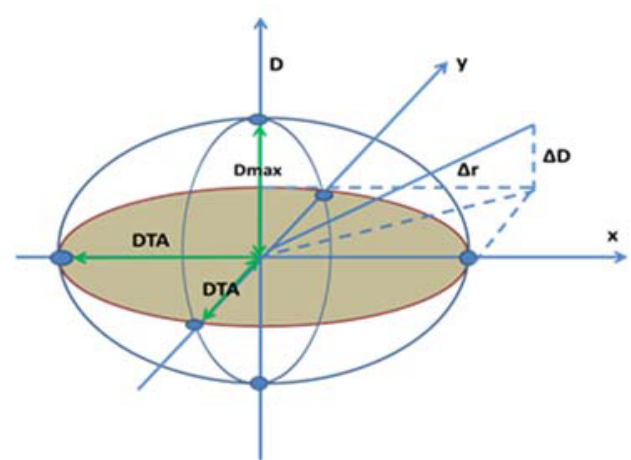

Figure 1. The principle of gamma verification: $x, y, D$ - spatial and dose dimensions; DTA; Dmax; $\Delta \mathrm{r}, \Delta \mathrm{D}$ - local spatial and dose divergence of the analyzed point

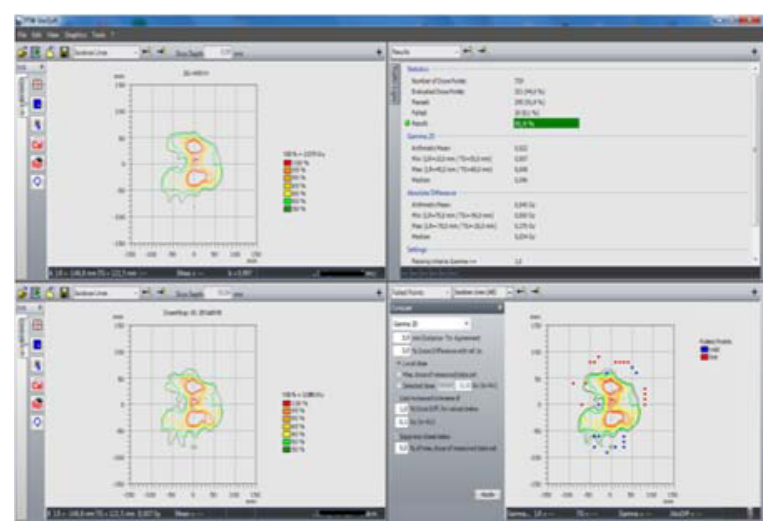

Figure 2. PTW VeriSoft 4.1

For gamma evaluation, dose fluence maps measured by $2 \mathrm{D}$-array and calculated by treatment planning system are required to compare. This comparison can be made with software. In our clinic, PTW VeriSoft 4.1 (PTW, Freiburg, Germany) used (Fig. 2). VeriSoft is software to load, evaluate and compare dose matrices. VeriSoft is used to compare measured dose matrices (e.g. 2DARRAY matrices or films) and corresponding calculated matrices (from treatment planning systems). The software can also be used for dose verification in IMRT (Intensity Modulated Radiation Therapy).

The gamma evaluation was made under the criteria of $3 \mathrm{~mm}$ DTA $-3 \%$ DD and $5 \mathrm{~mm}$ DTA - 5\% DD. The dose below $5 \%$ of maximum dose of measured data set was suppressed. Gamma results of both phantoms were compared each other.

\section{Results}

The results of $3 \mathrm{~mm}$ DTA - 3\% DD and $5 \mathrm{~mm}$ DTA $5 \%$ DD gamma analysis for RW3 slab phantom with all set-up parameters set to original angles and with all setup parameters set to $0^{0}$ are shown in Table 1 and Table 2 , respectively. Also, the results of $3 \mathrm{~mm}$ DTA $-3 \%$ DD and $5 \mathrm{~mm}$ DTA - 5\% DD gamma analysis for Octavius phantom with all set-up parameters set to original angles and with all set-up parameters set to $0^{0}$ are shown in Table 3 and Table 4, respectively.

Table 1. RW3 Slab Phantom: $3 \mathrm{~mm}$ DTA - 3\% DD and $5 \mathrm{~mm}$ DTA - 5\% DD Gamma Analysis: Parameters are set to original angles

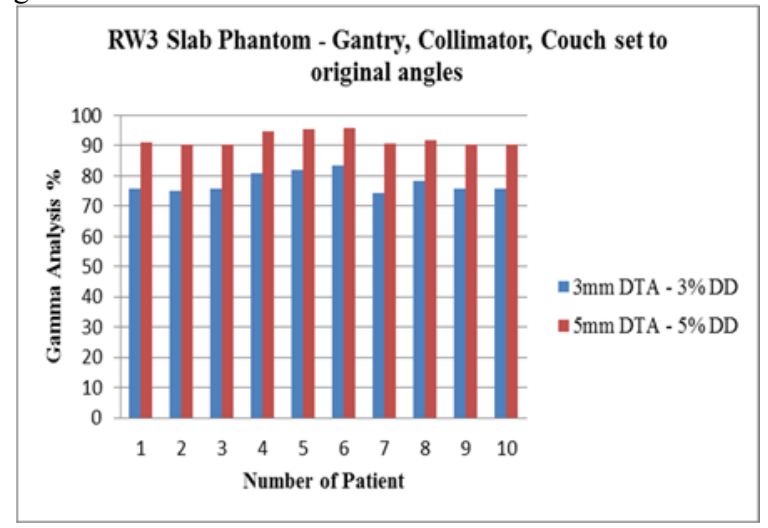

Table 2. RW3 Slab Phantom: $3 \mathrm{~mm}$ DTA - 3\% DD and $5 \mathrm{~mm}$ DTA - 5\% DD Gamma Analysis: Parameters are set to $0^{0}$

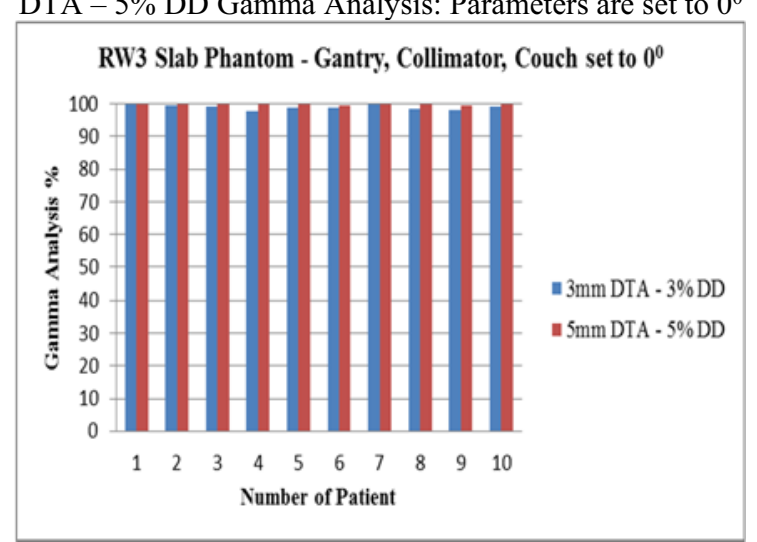


Table 3. Octavius Phantom: $3 \mathrm{~mm}$ DTA - 3\% DD and $5 \mathrm{~mm}$ DTA - 5\% DD Gamma Analysis: Parameters are set to original angles

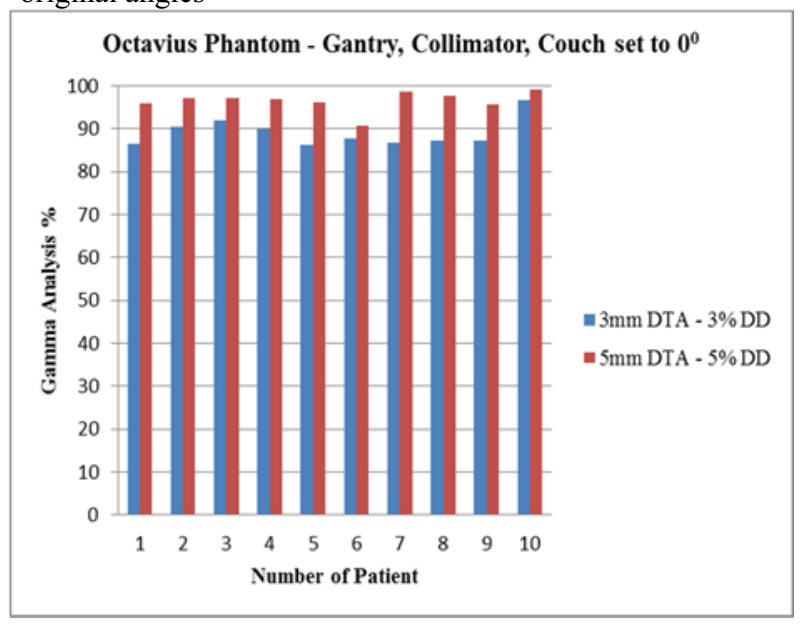

Table 4. Octavius Phantom: $3 \mathrm{~mm}$ DTA - 3\% DD and $5 \mathrm{~mm}$ DTA - 5\% DD Gamma Analysis: Parameters are set to $0^{0}$

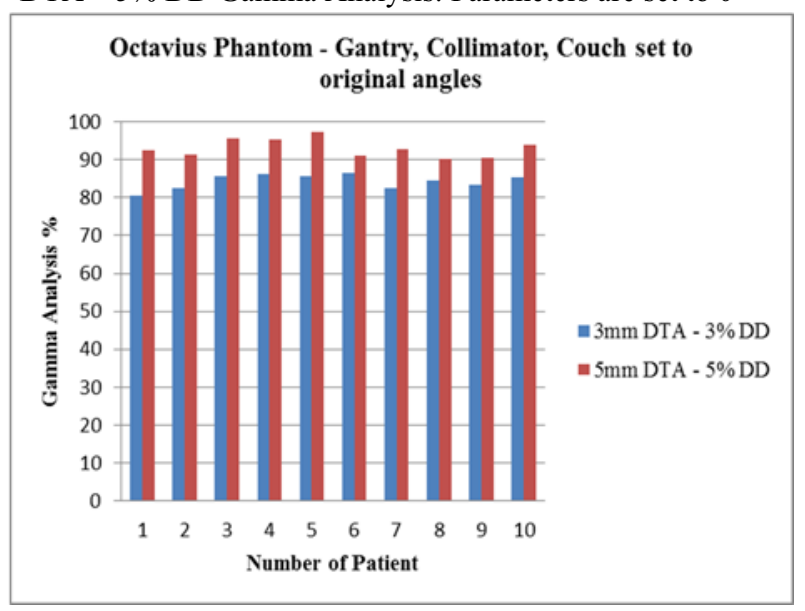

Mean value of $3 \mathrm{~mm}$ DTA - 3\% DD analysis for RW3 phantom with original set-up parameters was found $77.63 \%$; minimum and maximum values were found $74.3 \%$ and $83.4 \%$, respectively. Mean value of $5 \mathrm{~mm}$ DTA - 5\% DD analysis for RW3 phantom with original set-up parameters was found $92.03 \%$; minimum and maximum values were found $90.2 \%$ and $95.6 \%$, respectively.

Mean value of $3 \mathrm{~mm}$ DTA-3\% DD analysis for RW3 phantom with set-up parameters were set to $0^{0}$ was found $98.95 \%$; min and max values were found $97.9 \%$ and $99.7 \%$, respectively. Mean value of $5 \mathrm{~mm}$ DTA-5\% DD analysis for RW3 phantom with set-up parameters were set to $0^{\circ}$ was found $99.85 \%$; min and max values were found $99.6 \%$ and $100.0 \%$, respectively.

Mean value of $3 \mathrm{~mm}$ DTA-3\% DD analysis for Octavius phantom with original set-up parameters was found $84.25 \%$; min and max values were found $80.4 \%$ and $86.4 \%$, respectively. Mean value of $5 \mathrm{~mm}$ DTA $5 \%$ DD analysis for Octavius phantom with original set- up parameters was found $92.97 \%$; min and max values were found $90.2 \%$ and $97.2 \%$, respectively.

Mean value of $3 \mathrm{~mm}$ DTA-3\% DD analysis for Octavius phantom with set-up parameters were set to $0^{0}$ was found $89.09 \%$; min and max values were found $86.3 \%$ and $96.7 \%$, respectively. Mean value of $5 \mathrm{~mm}$ DTA-5\% DD analysis for Octavius phantom with setup parameters were set to $0^{\circ}$ was found $96.55 \%$; min and max values were found $95.7 \%$ and $99.2 \%$, respectively.

\section{Conclusion}

Radiation therapy is the main treatment modality for most of the head and neck cancer. Intensity modulated techniques have advantage of delivering maximum dose to target while protecting the normal tissues. Computed tomography scanning, immobilization, contouring the volumes of interest, treatment planning, set-up and dose delivery are the parts of treatment and every step of them extremely important. In IMRT technique, a successful dose delivery is strongly related to treatment planning. Treatment plans must be verified by QA plans before irradiation of the patient.

In this study, 10 nasopharyngeal carcinoma patients' treatment plans were used to create QA plans. $52^{\circ}, 104^{\circ}$, $156^{\circ}, 208^{\circ}, 260^{\circ}$ and $312^{\circ}$ angles were chosen for 7 fields. Dynamic leaf shaped fields make the study more meaningful, because the dose fluence verification becomes more important. For the gamma analysis, normalization of calculated and recorded dose matrixes was performed inside regions of homogeneous dose. Commercially different types of QA tools are available. In our clinic, we use RW3 slab phantoms and Octavius phantom for QA.

In all conditions of phantom set-up and gamma analysis criteria, 5mm DTA-5\% DD gave the highest values as expected. As we seen in tables above, setting set-up parameters to $0^{\circ}$ gave better results compare to original set-up parameters for both RW3 and Octavius phantoms. The best results were obtained from RW3 slab phantom by setting gantry, collimator and couch to $0^{\circ}$.

Spezi et al. [4], compared output factors with 2Darray and pinpoint chamber measurements and the results were coherent. Also, output factors agreed with reference dataset for field sizes ranging from $2 \times 2 \mathrm{~cm}^{2}$ to $27 \times 27 \mathrm{~cm}^{2}$. This can be considered a very good achievement since it is not trivial to obtain good output factor response for small radiation fields when using matrices of detectors. Studies reported the diodes of the 2D array used in IMRT verification have angular dependence which would lower the verification accuracy when the 2D array is used in measuring the actual beams of the treatment plan. Thus, all the beam gantry angles should be modified to $0^{\circ}$ for the verification of the IMRT treatment plan $[5,6]$. Li et al. [7], suggested that the $2 \mathrm{D}$ array can be used in the verification of the composite dose distribution of IMRT treatment plan if enough solid water slabs are attached 
around the $2 \mathrm{D}$ array and the beam incidence angles are not in the range of $90^{\circ}$ or $270^{\circ} \pm 5^{\circ}$. Chandraraj et al. [8], reported that when stricter gamma index criteria were used, some of the measured planar doses failed to pass the tolerance of $90 \%$.

IMRT is a modern accurate irradiation technology characterized by the highly conformal radiation dose to the planning target volume and great steep dose gradients [99]. We consider gamma evaluation method as a reliable and effective instrument for IMRT treatment plan verification. The method should be performed by $0^{\circ}$ angles of gantry, collimator and couch in RW3 slab phantoms with considering angular dependence of 2D-array detectors.

\section{References}

1. D.A. Low, J.M. Moran, J.F. Dempsey, L. Dong, M. Oldham. Med. Phys. 38, 1313 - 1338 (2011)

2. PTWFreiburg; http://www.ptw.de/imrt imat_octavius.html?\&cId=4 160

3. D.A. Low, W.B. Harms, S. Mutic, J.A. Purdy. Med. Phys. 25, 656 - 661 (1998)

4. E. Spezi, A.L. Angellini, F.Romani, A. Ferri. Phys. Med. Biol. 50, 3361 - 3373 (2005)

5. D. Letourneau, M. Gulam, D. Yan, M. Oldham, J.W. Wong. Radiother. Oncol. 70, 199 - 206 (2004)

6. B. Fraass, K. Doppke, M. Hunt, G. Kutcher, G. Starkschall, R. Stern, J.V. Dyke. Med. Phys. 25, 1773 - 1829 (1998)

7. Q.L. Li, X.W. Deng, L.X. Chen, X.Y. Huang, S.M. Huang. Chin. J. Cancer 29, 617 - 620 (2010)

8. V. Chandraraj, S. Stathakis, R. Manickam, C. Esquivel, S. Supe, N. Papanikolaou. J. Appl. Clin. Med. Phys. 12, 338 - 349 (2011)

9. Y.M. Hu. Radiation Oncology Physics. Beijing: Atomic Press, 538-541 (1999) 